\title{
SOLAR TRACKER PLATFORM DEVELOPMENT FOR ENERGY EFFICIENCY IMPROVEMENT OF PHOTOVOLTAIC PANELS
}

\author{
CSABA SZÁSZ \\ Department of Electrical Machines and Drives, Technical University of Cluj, Cluj, Romania \\ E-mail: Csaba.Szasz@emd.utcluj.ro
}

\begin{abstract}
Solar energy systems have emerged over the last decades as the cleanest and most abundant renewable energy resources available worldwide. Solar trackers are devices specially developed to enhance the energy efficiency of solar energy systems. This paper presents the design and implementation stages of a reconfigurable hardware technology-based two-axis solar tracker platform, specially conceived to improve the energy efficiency of photovoltaic (PV) panels. The main module of this platform is the NI MyRIO ready-to-use development system built upon a high-performance Field Programmable Gate Array (FPGA) processor that controls the entire solar tracker unit. Optimal tracking of the sun movement and obtaining the maximal energy efficiency rate is achieved by simultaneous real-time controlling both the captured sunlight intensity and PV cell temperature magnitudes. In this way, a robust and versatile positioning system has been developed that performs a high precision and accurate tracking pathway. All the control algorithms are implemented there under the LabView graphical programming software toolkit. The final solution boosts in a useful and modularized tracking system that looks useful in a wide range of applications both in industrial and domestic project sites with different power scales.
\end{abstract}

Keywords: tracking system, energy efficiency, reconfigurable technology, FPGA processor, PV panel

\section{Introduction}

It is well known that solar PV modules and panels work on their maximal energy conversion rate when their absorbing surface area remains perpendicular to the incoming sun rays. While static PV panels remain physically fixed in their surrounding environment, their mobile counterparts continuously are oriented directly to the suns radiant energy, in order to capture the maximal sun light intensity and achieve optimal energy transfer benefit. In fact, solar trackers are positioning systems for "orientating" panels toward the actual position of the sun. The challenge in getting the maximum solar power conversion efficiency is to ensure that the complete PV array (or module) is optimally oriented with regards to the incoming sunlight all day long and every day of the year. Obviously, the ideal solar tracker platform is the one mounted along the equatorial latitude, in a similar way that the high performance telescopes or complex satellite communication antennas are placed. Unfortunately, such ideal solutions would be very expensive and also un- reachable for most of the industrial and domestic customers. However, it is no doubt that nowadays solar trackers are rising in popularity and represent highly accepted solutions in a wide range of solar energy conversion applications. In this circumstance it looks not without importance to take a short survey and evidence their major advantages and drawbacks in concrete situations.

First of all, among the major advantages of solar trackers may be mentioned as follows [1]:

- the energy conversion rate of a given size PV module used in a solar tracker mounting configuration is significantly higher (with around 10 $20 \%$ ) than its corresponding fixed-mount counterpart with the same surface or array;

- they are ideal for optimizing land usage because they produce more energy than the fixed-mount solution ones in the same space;

- maintenance issues related to tracking systems have been greatly reduced thanks to the important advancements reached in mechanics, electronics and digital system's reliability.

Open Access statement. This is an open-access article distributed under the terms of the Creative Commons AttributionNonCommercial 4.0 International License (https://creativecommons.org/licenses/by-nc/4.0/), which permits unrestricted use, distribution, and reproduction in any medium for non-commercial purposes, provided the original author and source are credited, a link to the CC License is provided, and changes - if any - are indicated. 
Beside all the above ranked, solar trackers also share important shortcomings, such as:

- they are more complex electromechanical systems than their fixed-mount counterparts;

- they are more expensive than their stationary counterparts;

- tracking systems accommodate with more difficulty to harsh environments in comparison with the fixed-mount solar PV panel systems;

- they require higher supervising and service (cleaning, conservation and maintenance) than the fixed-mount similar devices.

In spite of all these advantages/disadvantages mentioned, solar tracker systems emerge as a well-fitted solution for a wide range of industrial and domestic applications, ranging from microwatt order even to megawatt order installed power sites.

\section{Solar tracker systems theoretical background}

In essence, solar tracking systems currently being used are divided into two main categories: single-axis tracking systems and dual-axis solar tracking systems. In the first case, solar PV panels can turn around their central axis (tracking the East-West solar motion, $E-W)$, the second ones have two independent rotation axes that allow them a two-degree of freedom and orientate as a mirror that captures the solar light in a perpendicular incidence. In this last situation both the East-West and South-North $(S-N)$ axis motion is performed at the same time. Such systems in international references are also named as solar panel azimuth- zenith orientation systems [2]. For better understanding of the above used terms, let us consider the simple explications given next in Fig. 1.

According to Fig. 1, the azimuth is the compass angle of the sun as it moves through the sky from $E$ to $W$ during the 24 hours of the day. The zenith is the angle between local zenith vector and the position of the sun in the sky. The entire solar panel orientation strategy relies upon these two values.

In the related international references often is mentioned that solar tracking systems perform in essence two main operations at the same time: solar panel rotation and solar panel tilt [2]. Solar panel orientation refers to the azimuth angle setting during the day. Therefore, for orientation of the PV platform it should be turned towards the terrestrial equator line and the panel will catch the greatest amount of solar radiation.

In a similar way, solar panel tilt refers to the zenith angle setting. Results that such kind of a solar tracker system should operate according to the basic principle exposed before in Fig. 2. This is in fact a dual-axis solar tracking system allowing a two-degree of freedom to orientate the PV panel or module. In this way the solar radiation may be captured in a perpendicular incidence and the platform can operate on its maximal energy conversion rate achieving its highest efficiency possible.

On the other hand, it is well known that the general efficiency of a PV module also depends on variables such as the ambient temperature $\left(T_{A}\right)$, the cell operation temperature $\left(T_{C}\right)$, or material properties. In Ref. [3] this interdependence is expressed with the mathematical relationship:

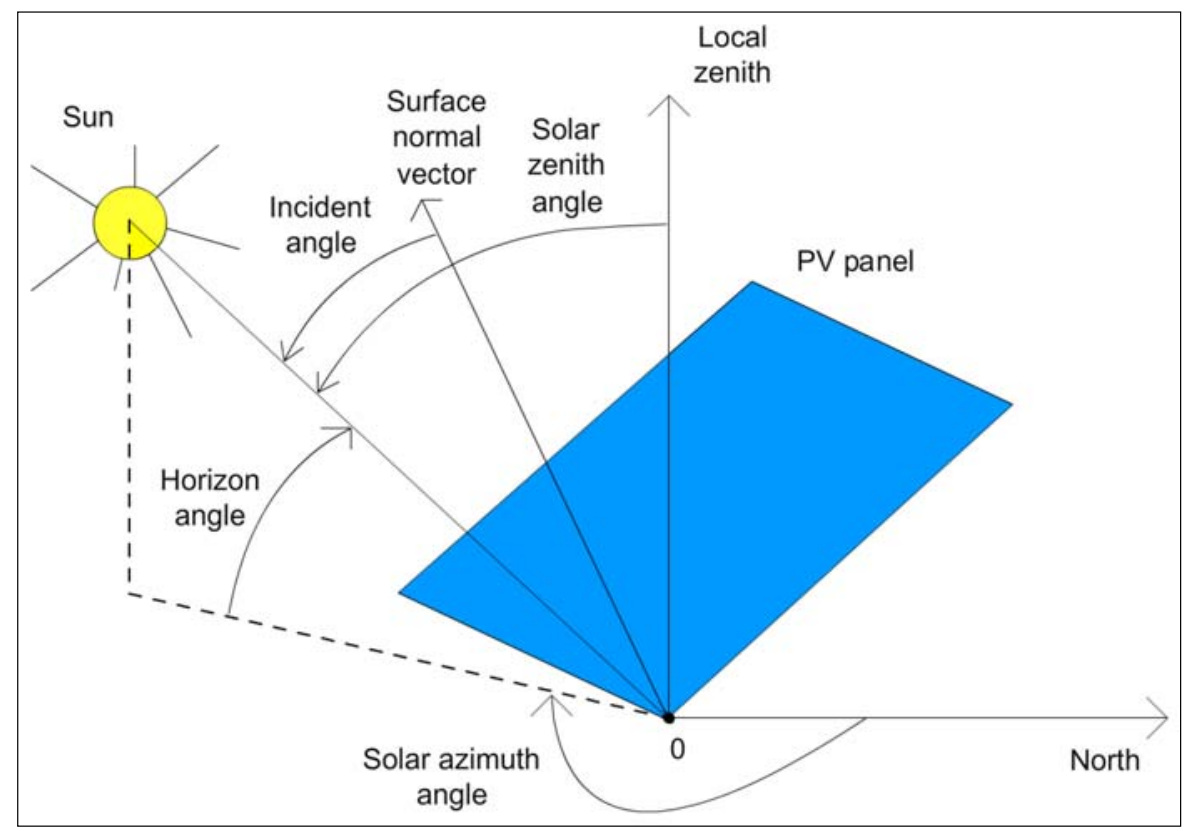

Fig. 1. Definition of the azimuth and zenith angles in solar orientation systems 


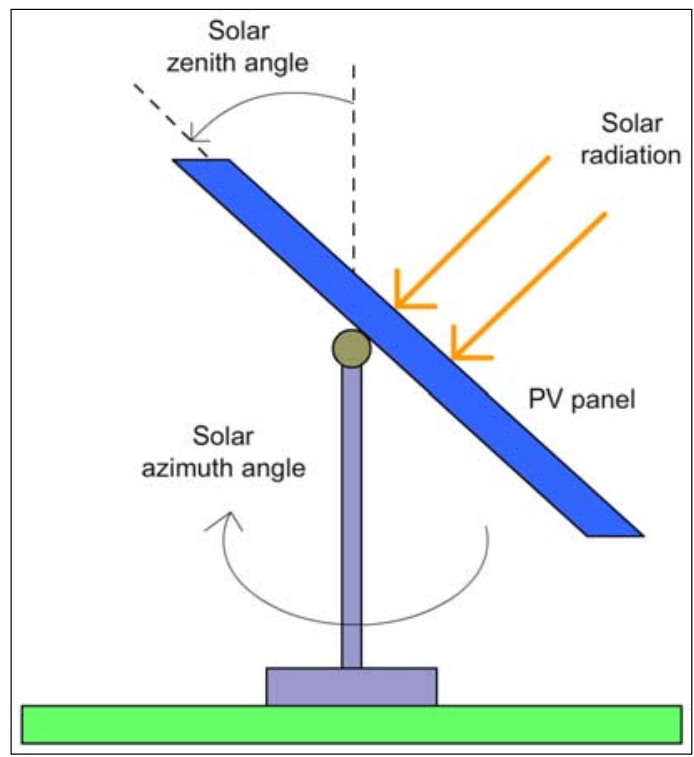

Fig. 2. Solar tracker system operation principle

$$
\eta=\eta_{r}\left[1-\beta_{r}\left(T_{C}-T_{r}\right)+\log _{10} G(t)\right] .
$$

In this formula $\eta_{r}$ means the energy conversion efficiency at standard test conditions (STC: $G_{r}=$ $1000 \mathrm{~W} / \mathrm{m}^{2}, T_{r}=25^{\circ} \mathrm{C}$ ), the temperature coefficient $\beta_{r}$ usually is given by the PV manufacturer, and $\gamma$ is a material coefficient. The temperature coefficient is given by the equation:

$$
\beta_{r}=\left[T_{0}-T_{r}\right]^{-1}
$$

where $T_{0}$ is the high temperature at which the conversion efficiency drops down to zero [4]. For variations of ambient temperature and irradiance intensity it is considered the empirical relationship which determines the operating temperature of the module $[5,6]$ :

$$
T_{C}=[1 / 0.8] \cdot\left(T_{N}-20\right) G+T_{A},
$$

where $T_{A}$ represents the ambient temperature $(K)$ and $T_{N}$ is the nominal operating cell temperature $(K)$ given in the manufacturer's datasheet. Results that:

$$
\begin{aligned}
\eta=\{1 & -\beta_{r}\left[T_{A}-T_{r}+\left(T_{N}-20\right) \cdot\left(G(t) / G_{r}\right)\right] \\
& \left.+\gamma \log _{10} G(t)\right\} \cdot 100 .
\end{aligned}
$$

In most of cases authors consider $\beta_{r}=0.0045 \mathrm{~K}$, $T_{N}=45{ }^{\circ} \mathrm{C}$, and $\eta_{r}$ is given as a catalogue data. Equation (4) defines completely the PV module energy conversion efficiency as function of the ambient temperature and solar irradiation intensity variations. Results that in order to achieve the highest efficiency rate is not sufficient to capture only the maximal solar radiation intensity, it is recommended also to measure the ambient temperature. This last observation lead to the conclusion that maybe it is better to con-

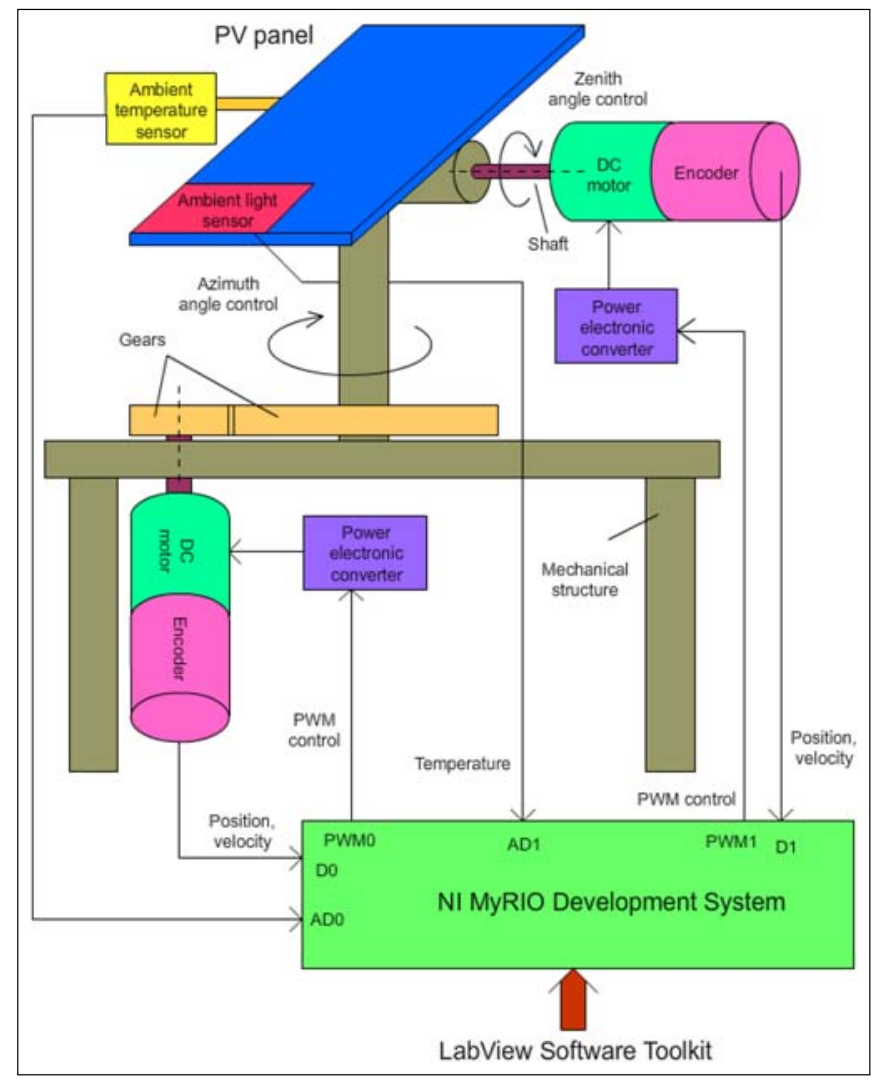

Fig. 3. The solar tracker platform block diagram 
ceive a two-axis solar tracking system that operates as function of two external variables: the solar radiation intensity $G(t)$ and the ambient temperature $T_{A}$. Then, once these two magnitudes have been measured may be found the maximal value of the conversion efficiency. In other words, it is better to maximize the energy conversion rate as function of both these two variables.

\section{The solar tracker platform development}

Considering the theoretical remarks unfolded in the previous paragraph, it is proposed a two-axis solar tracker platform as is presented in the block diagram shown next in Fig. 3. There is plotted a specially conceived mechanical structure that supports the PV panel selected for experimental measurements and laboratory test operations.

The solar tracking orientation of the PV module is performed by using two DC motors that ensure the optimal azimuth and zenith angle control (Fig. 4).

These are in fact DC motor/Gearbox units with rugged, heavy duty construction that includes incremental encoder for sensing rotation and speed [3]. The gear ratio is 1:19 and the motors may be supplied with custom voltage up to $12 \mathrm{~V}$. Figure 4 shows a pic-

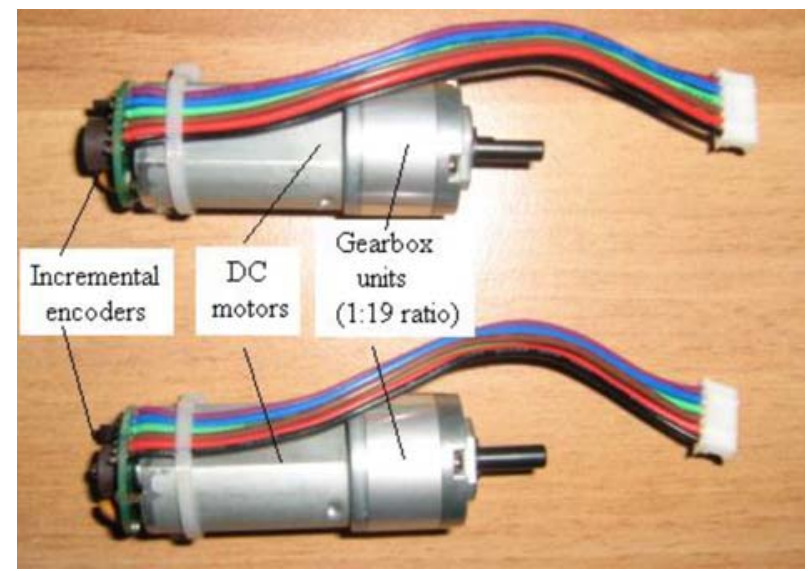

Fig. 4. The DC motors used for azimuth and zenith angle control

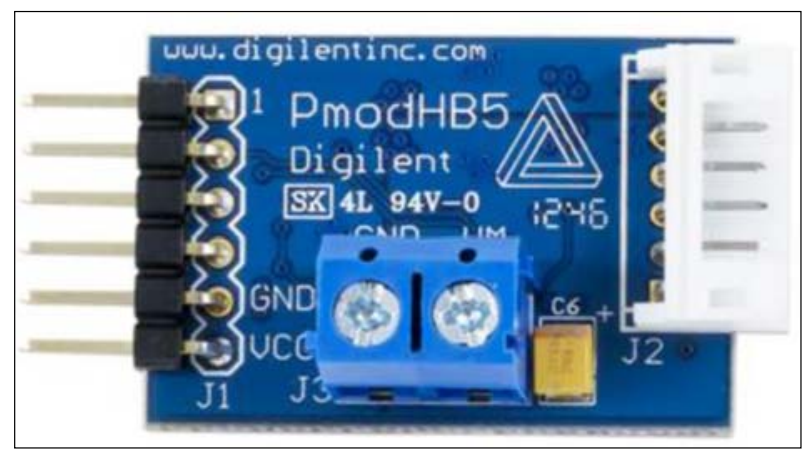

Fig. 5. The Pmod HB5 converter PCB for DC motors drive [8] ture that evidences the electromechanical construction of these two modularized DC motors. They are driven by two stand-alone Digilent Co. made Pmod HB5type power electronic converters [8].

The HB5 converter is in fact a $2 \mathrm{AH}$-bridge circuit for small to medium size DC motors drive. The PCB also include two sensor feedback pins to capture position/velocity information from the DC motor/gearbox, respectively other two pins to enable the $\mathrm{H}$-bridge full operation and to set up the servomotor rotation sense [8]. The solar radiation intensity is measured by using the Pmod ALS sensor that is physically mounted right on the surface of the analyzed PV panel [9].

The Digilent Co. Pmod ALS converts light intensity into digital information with 8-bit resolution by using the ADC081S021 analog to digital converter IC. The board communicates with a host board via the serial-parallel interface protocol. It is delivered a single reading in a 16 SCLK clock cycles (Pmod ALS requires the frequency of SCLK between 1-4 MHz) and the location of this communication line is marked on the Pmod header. The recommended optimal supply voltage of the ALS sensor is $3.3 \mathrm{~V}$ [9]. Next, for the ambient temperature measurement the Pmod TMP3 digital sensor is used that is mechanically fixed near to the PV module [10].

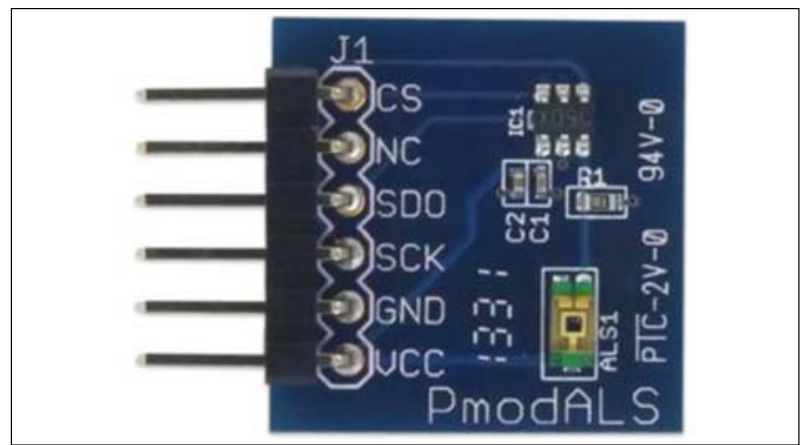

Fig. 6. The Pmod ALS ambient light sensor PCB [9]

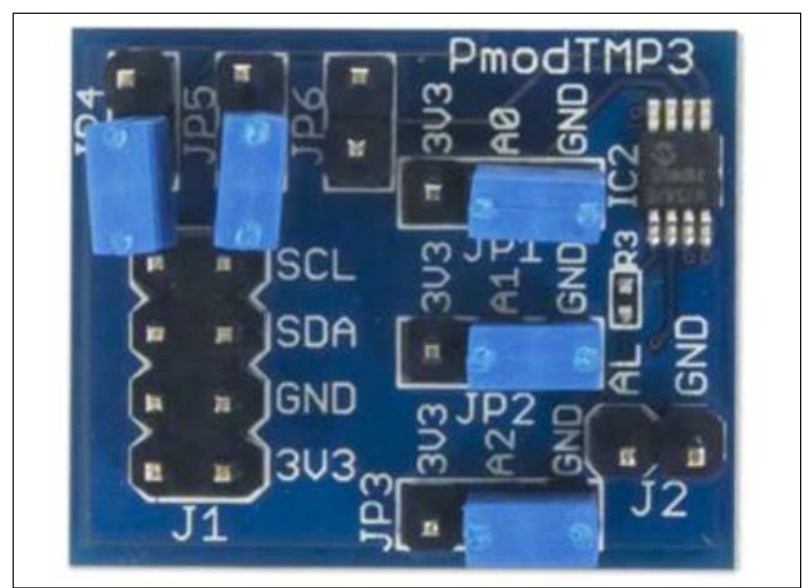

Fig. 7. The Pmod TMP3 temperature sensor PCB [10] 
The Digilent Pmod TMP3 temperature sensor has been built around the TCN75A integrated circuit that provides users with up to 12 bits of resolution information about its environment temperature. The typical accuracy of this circuit is $\pm 1^{\circ} \mathrm{C}$, with a maximal resolution of $0.0625^{\circ} \mathrm{C}$ and $30-240$ ms conversion time. The TMP3 communicates with a host board via the well-known $\mathrm{I}^{2} \mathrm{C}$ protocol. The serial clock and serial data lines need to be pulled up to the logic high voltage level through pull-up resistors [10].

Finally, the information captured from the Pmod ALS and Pmod TMP3 sensors is acquired by the host board NI MyRIO Development Kit. This is a readyto-use compact embedded system manufactured by the National instruments Co. that provides analog input, analog output, digital input/output and audio channels to communicate with external devices [11]. The analog input channels are placed on MyRIO expansion ports $A$ and $B$, on connector $C$ and a stereo audio input connector. All these input channels are multiplexed to a simple analog-to-digital converter. The analog output channels are placed on the same connectors. Each one has a dedicated digital-to-analog converter therefore they allow simultaneous digital/ analog conversions. Toward, the NI MyRIO development system provides 16-bit digital input/output lines both on the $A$ and $B$ connectors, UART port, buttons, status and user LEDs, four PWM channels for motor control purposes, respectively three embedded accelerometers [11]. In accordance with the implementation strategy plotted in Fig. 3, the temperature and light intensity information are captured by using the analog input channels of the MyRIO system, the two DC motors will by driven via the available PWM channels and the position and velocity information from the encoders are acquired through digital input lines. In this way a versatile and high performance embedded digital system may be implemented for real-time control of the solar tracker platform.

\section{Experimental implementation}

Obviously, the design and development steps presented in the previous paragraph have been followed by implementation and test operations. Figure 8 presents the laboratory setup built-up to experiment the solar tracker platform prototype.

There it is possible to identify the NI MyRIO development board that is interfaced with the rest of components of the tracking system. The TMP3 temperature sensor has been wired to the $A$ connector of the board, the Pmod ALS ambient sensor to the $B$ connector and the Pmod HB5 inverter units to each one of the above two expansion connectors. The power converter modules drive the two DC motors of the azimuth and zenith angles control. A full picture of the entire development setup for laboratory experiments and test operations is shown next in Fig. 9. In this situation the digital system communicates with a host PC computer on which has been developed the full control algorithm under the LabView graphical software programming platform. The two DC motors are supplied by using a $12 \mathrm{~V}$ power unit.

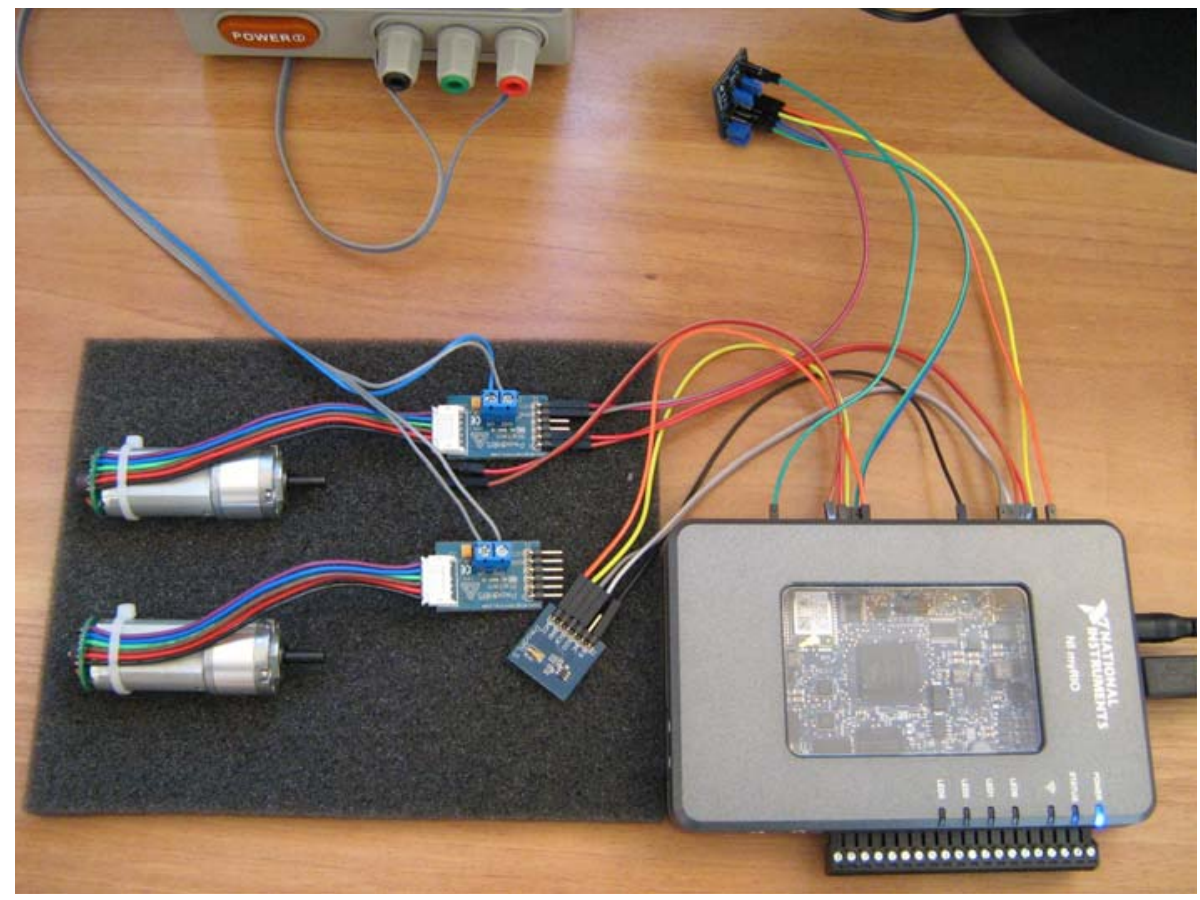

Fig. 8. The NI MyRIO development kit interfaced with the temperature and ambient light sensors and two DC motors 


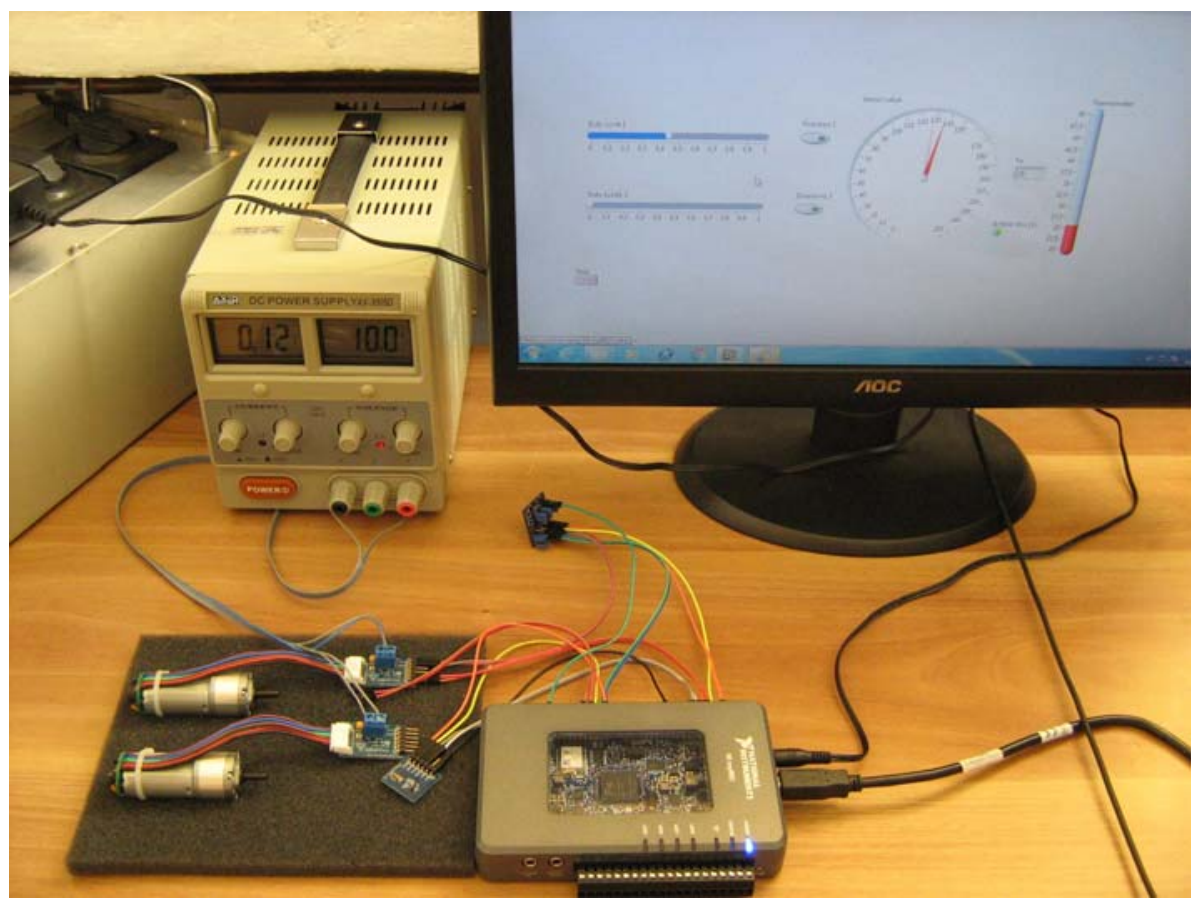

Fig. 9. The laboratory setup for test operations and experiments

On the PC screen may be observed the LabView front panel of the application that controls the solar tracker platform. There it is plotted an indicator that graphically shows the variation of the measured ambient temperature, one other indicator that plots the instantaneous value of the acquired ambient light intensity. Two slide-switches adjust the duty cycle of the generated PWM pulses that control the DC motors velocity respectively two user buttons set the rotation sense of these servomotors. A more detailed view of the above discussed control panel implemented as a virtual instrument (VI) is presented in Fig. 10.

The corresponding block diagram of the VI is plotted next in Fig. 11. There are evidenced the standard VI component blocks implemented on the digital control system. For example, the two PWM blocks ( $P W M 1$ and $P W M 2$ ) provide the constant frequency but variable duty cycle pulse trains for the DC motors drive. Their rotation sense has been set previously via the $A / D I O 7$ and $B / D I O 7$ digital output lines. In a similar way, the information corresponding to the instantaneous ambient temperature is captured by using the $I 2 C$ standard VI for serial communication that has been programmed with the constant values prescribed in the catalogue datasheet of the used TCN75A IC. After same minor adjustments the acquired information becomes suitable for plot in decimal ${ }^{\circ} \mathrm{C}$ units. The SPI (Serial Parallel Interface) VI ensures the high precision measurement of the ambient light intensity and plots the processed information on an adequate

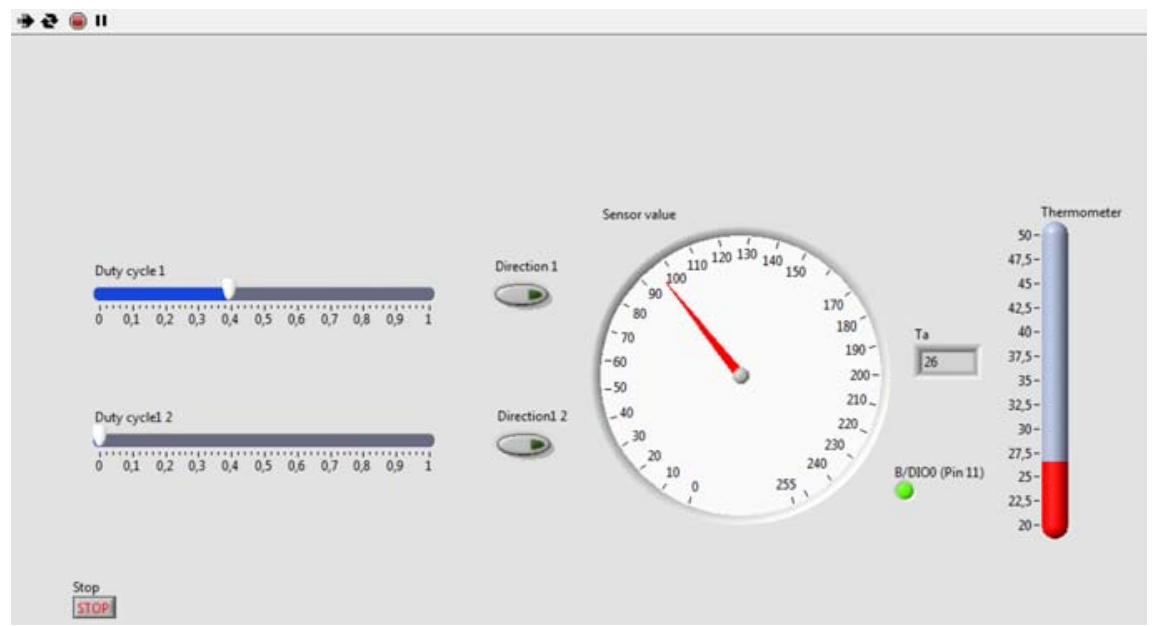

Fig. 10. The LabView front panel of the VI developed for the solar tracking platform control 


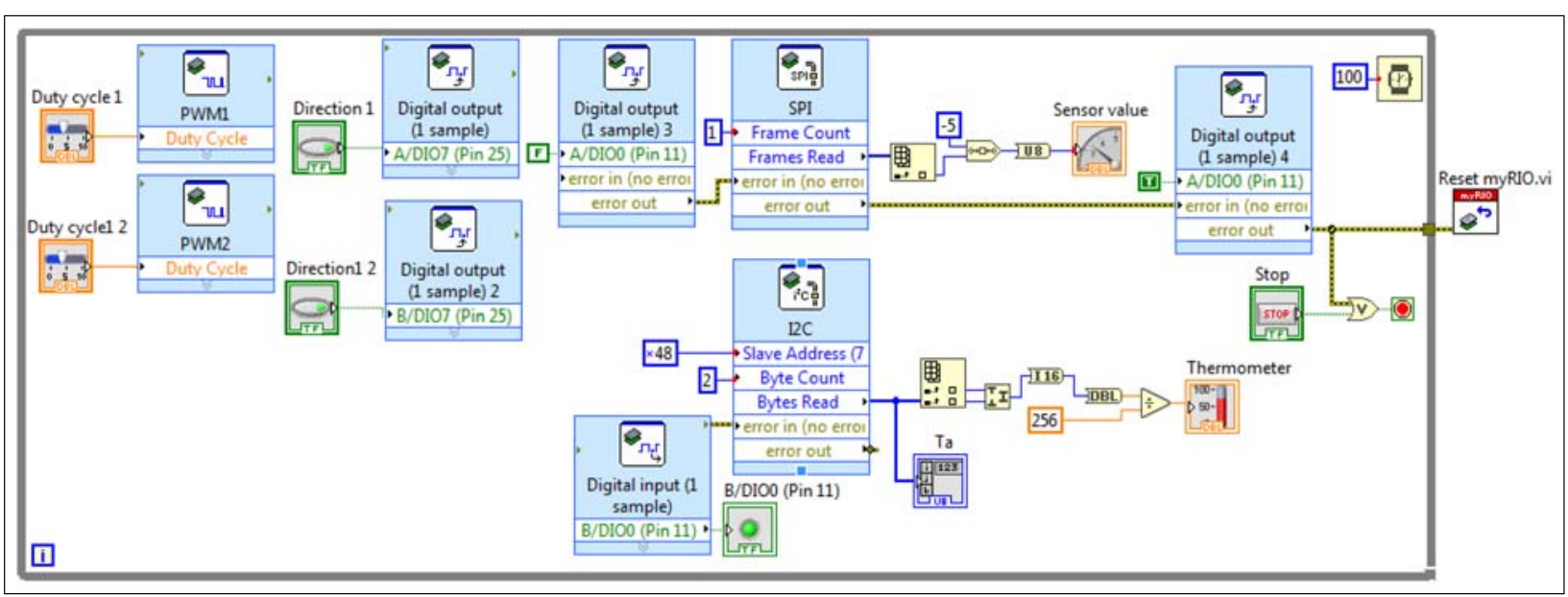

Fig. 11. The LabView block diagram of the VI developed for the solar tracking platform control

rotational indicator. If any error occurs during all these measurement operations the NI MyRIO board will be turned on into its initial reset state.

However, entire solar tracking system is still under full development. The presented program operates properly but is missing yet some calibration and precision adjustment tasks, or measurements regarding the PV panel overall energy efficiency. Therefore the presented program is not finished yet and is under a continuous development and improvement process. Additionally a concrete algorithm for precise tracking of the sun movement in the sky is waiting for implementation and test operations. In spite of all these shortcomings, the presented and implemented solar tracking platform looks an efficient and high reliability embedded system for researches and studies that targets the energy efficiency improvement of modern solar PV panels or modules.

\section{Conclusions}

In international references plenty of high quality research is dedicated to solar energy transformation processes in renewable electrical energy. Between these an important amount is focused on improving energy efficiency of PV panels or modules. This paper presents the development steps of a reconfigurable hardware technology-based two-axis solar tracker platform specially conceived to improve solar PV panel's energy efficiency. The main idea is that both the ambient temperature and ambient light intensity information simultaneous real-time control is necessary to reach the highest energy efficiency rate of a photovoltaic module. The solution to use the ready-to-use NI MyRIO development kit with the adequate standard interface modules looks a proper choice in order to achieve a high performance and versatile embedded solar tracking control system. This setup provides high reliability and accurate tracking, respectively looks useful in a wide range of applications both in industrial and domestic project sited with different power scales. The presented solution is also recommended as a useful system for researchers involved in studies related to improve PV panels efficiency and solar energy conversion rate.

\section{References}

[1] https://www.solarpowerworldonline.com/2016/05/ advantages-disadvantages-solar-tracker-system/

[2] http://www.alternative-energy-tutorials.com/solar-power/ solar-panel-orientation.html

[3] Fesharaki V. J., Dehghasi M., Fesharaki J. J. (2011), The effect of temperature on photovoltaic cell efficiency. In: Proceedings of the 1st International Conference on Emerging Trends in Energy Conversion, Teheran, Iran, 20-21 November 2011, pp. 1-6.

[4] Evans D., Florschuetz L. W. (1978), Terrestrial concentrating photovoltaic power system studies. Sol. Energ., 20, $37-43$.

[5] Velkovski B., Pejovski D. (2016), Application of incremental conductance MPPT method for a photovoltaic generator izn LabView. In: 20th International Student Conference on Electrical Engineering, 24th May 2016, Prague, pp. 1-6. (Poster)

[6] Jaleel J. A., Omega A. R. (2012), Simulation on maximum power point tracking of the photovoltaic module using LabView. International Journal of Advanced Research in Electrical, Electronics and Instrumentation Engineering, 1(3), 190-198. ISSN 2278-8875

[7] https://store.digilentinc.com/motor-gearbox-1-19-gearratio-custom-12v-motor-designed-for-digilent-robot-kits/

[8] https://store.digilentinc.com/pmod-hb5-h-bridge-driverwith-feedback-inputs/

[9] https://store.digilentinc.com/pmod-als-ambient-lightsensor/

[10] https://store.digilentinc.com/pmod-tmp3-digitaltemperature-sensor/

[11] http://www.ni.com/ro-ro/support/model.myrio-1900.html 